\title{
Protocol for regional implementation of collaborative self-management services to promote physical activity
}

\author{
Anael Barberan-Garcia ${ }^{1,2^{*}}$ (D) Elena Gimeno-Santos ${ }^{1,2}$, Isabel Blanco ${ }^{1,2}$, Isaac Cano², Graciela Martínez-Palli1 ${ }^{2,3}$, \\ Felip Burgos ${ }^{1,2}$, Felip Miralles ${ }^{4}$, Miquel Coca ${ }^{3}$, Serafín Murillo ${ }^{5}$, María Sanz ${ }^{2,6}$, Alexander Steblin ${ }^{4}$, Marta Ubré ${ }^{3}$, \\ Jaume Benavent ${ }^{7}$, Josep Vidal ${ }^{5}$, Marta Sitges ${ }^{2,6}$ and Josep Roca ${ }^{1,2^{*}}$
}

\begin{abstract}
Background: Chronic diseases are generating a major health and societal burden worldwide. Healthy lifestyles, including physical activity (PA), have proven efficacy in the prevention and treatment of many chronic conditions. But, so far, national PA surveillance systems, as well as strategies for promotion of PA, have shown low impact. We hypothesize that personalized modular PA services, aligned with healthcare, addressing the needs of a broad spectrum of individual profiles may show cost-effectiveness and sustainability.

Methods: The current manuscript describes the protocol for regional implementation of collaborative selfmanagement services to promote PA in Catalonia (7.5 M habitants) during the period 2017-2019. The protocols of three implementation studies encompassing a broad spectrum of individual needs are reported. They have a quasiexperimental design. That is, a non-randomized intervention group is compared to a control group (usual care) using propensity score methods wherein age, gender and population-based health risk assessment are main matching variables. The principal innovations of the PA program are: i) Implementation of well-structured modular interventions promoting PA; ii) Information and communication technologies (ICT) to facilitate patient accessibility, support collaborative management of individual care plans and reduce costs; and iii) Assessment strategies based on the Triple Aim approach during and beyond the program deployment.
\end{abstract}

Discussion: The manuscript reports a precise roadmap for large scale deployment of community-based ICT-supported integrated care services to promote healthy lifestyles with high potential for comparability and transferability to other sites.

Trial registration: This study protocol has been registered at ClinicalTrials.org (NCT02976064). Registered November 24th, 2016.

Keywords: Chronic disorders, eHealth, Integrated care, Physical activity, Cardiopulmonary rehabilitation, Service adoption

\section{Background}

Physical inactivity is acknowledged as a significant public health problem worldwide with kernel implications on major chronic conditions $[1,2]$. On the other hand, the positive effects of physical activity (PA) on health outcomes have been extensively demonstrated both in chronic

\footnotetext{
* Correspondence: anbarber@clinic.cat; jroca@clinic.ub.es

${ }^{1}$ Respiratory Medicine Department, Hospital Clínic de Barcelona, CIBERES,

Barcelona, Catalonia, Spain

Full list of author information is available at the end of the article
}

patients $[3,4]$ and in subjects at risk for developing chronic diseases [5-10].

The growing awareness on the health burden generated by insufficient levels of PA has prompted the interest for deploying community-based initiatives aiming at fostering active healthy living [11]. However, none of the interventions evaluated so far have reached large scale adoption. Moreover, traditional cardiopulmonary rehabilitation interventions temporary enhance aerobic capacity [12-14]; but, they show transient effects, rather low accessibility and no impact on behavioral changes resulting in enhanced PA. In

(c) The Author(s). 2018 Open Access This article is distributed under the terms of the Creative Commons Attribution 4.0 International License (http://creativecommons.org/licenses/by/4.0/), which permits unrestricted use, distribution, and 
contrast, different small trials seem to suggest efficacy of flexible modular service approaches (i.e. walking, urban training, pedometer-based program, tele-coaching) aiming at promoting active lifestyles [15-19]. Likewise, the analyses of the impact of information and communication technologies (ICT) on healthcare point out their role as enabling tools to support innovative integrated care services through: i) Empowerment of patients for self-management involving adoption of healthy life styles; and, ii) Promotion of efficient interactions among patients, caregivers, community services and healthcare professionals. Thus, ICT-supported services facilitate: i) Patient accessibility; ii) Health coaching; and, iii) Remote follow-up and incentives for patient's behavioral change [20].

The current protocol relies on the hypothesis that properly tailored self-management services, fully integrated in patient's health action plan with remote off-line professional support, may induce sustained behavioral changes resulting in active lifestyle behavior. A recent study [21] assessing barriers to deployment of such a collaborative self-management services was useful to identify the requirements for large scale deployment of the novel approach. Accordingly, the current study protocol aims to address those unmet requirements, namely: i) Workflow design of the PA services engaging both patients and healthcare professionals; ii) Enhanced ICT-support; iii) Evaluation strategies including structured indicators during and beyond deployment of the novel services; and, iv) Implementation of innovative business models.

The ultimate aim of the novel PA services for chronic patients and citizens at risk is to generate sustained enhancement of PA with health value generation. Hence, is expected a positive impact on multi-morbidities in terms of: i) Preventing occurrence, ii) Reducing exacerbations, and, iii) Modulating disease progress. The main outcome of the current protocol will be a roadmap for large-scale deployment and assessment of novel collaborative self-management PA services in the region of Catalonia (7.5 million citizens). The approach taken for promotion of PA shall have potential for generalization to other interventions aiming at promoting healthy lifestyles, as part of regional integrated care strategies for chronic patients.

\section{Methods/design}

\section{Setting}

The protocol has been designed as part of the 2016-2020 Health Plan in Catalonia [22] that involves regional deployment of integrated care services. It has been conceived as a 24 months test bed period plus a third year wherein the initiative will be deployed at regional level. At the end of the second year (2018), three main achievements will be in place. Firstly, the three implementation studies depicted in Fig. 1 will be adopted as mainstream services in one of the four healthcare sectors of the city of
Barcelona, Barcelona-Esquerra (AISBE) (540.000 inhabitants). A second milestone will be the elaboration of a plan for generalization of the PA services approach to other non-pharmacological interventions. Thirdly, a roadmap for regional deployment of the PA services in Catalonia (7.5 million habitants) will be launched.

The technological support used in the studies facilitates collaborative work among different healthcare professionals as well as patient empowerment for self-management. This scenario involves deployment of a Digital Health Framework [23, 24] bridging formal and informal care through adoption of a modified version of the current regional personal health folder (PHF) (La Meva Salut ${ }^{\oplus}$ LMS) (https:// lamevasalut.gencat.cat/web/guest/pre-login-cps) (Catalonia) as a self-management tool adopted to facilitate patient empowerment for self-care and enhanced monitoring. Moreover, the PHF is linked to the regional shared electronic health record $\left(\mathrm{HC}^{3}\right)$ [25], and provides citizens with an access point to their health information (i.e. discharge reports, imaging, electronic prescription, etc.), medical appointments and potentially for informal health data sources (e.g. mobile health applications, community-based social support services, etc.) as displayed in Fig. 1.

Another key ICT-supporting tool for the current PA protocol is an adaptive case management (ACM) system [26] to support collaborative work among case managers and to facilitate adaptive changes to the continuously evolving needs of the patients within well-structured service workflows. The ACM system will provide support to selection and scheduling of specific tasks throughout the clinical process, as well as facilitation of ad-hoc collaborations with other professionals across healthcare and social support tiers. The ACM platform will be open source and built-up on top of the current health information systems of the different healthcare providers and it will use existing regional interoperability infrastructures alluded to above, such as the shared $\mathrm{HC}^{3}$ and the PHF.

Large scale deployment of the current protocol is supported by convergence of different types of resources from: i) Institutional bodies (Department of Health, Generalitat de Catalunya); ii) Healthcare providers (Hospital Clinic de Barcelona); and, iii) EU Grants (see funding section of the manuscript). The Ethical Committee for Clinical Research at Hospital Clinic de Barcelona approved the study $(\mathrm{HCB} / 2016 / 0883)$ which has been registered at ClinicalTrials.org (NCT02976064). Patients' acceptance and signature of the informed consent will be required to participate in all the studies.

\section{Aims of the implementation studies}

The protocol addresses the four aims displayed in Fig. 2. Firstly, implementation of three collaborative self-management services designed to promote PA in three study groups representative of different layers of 


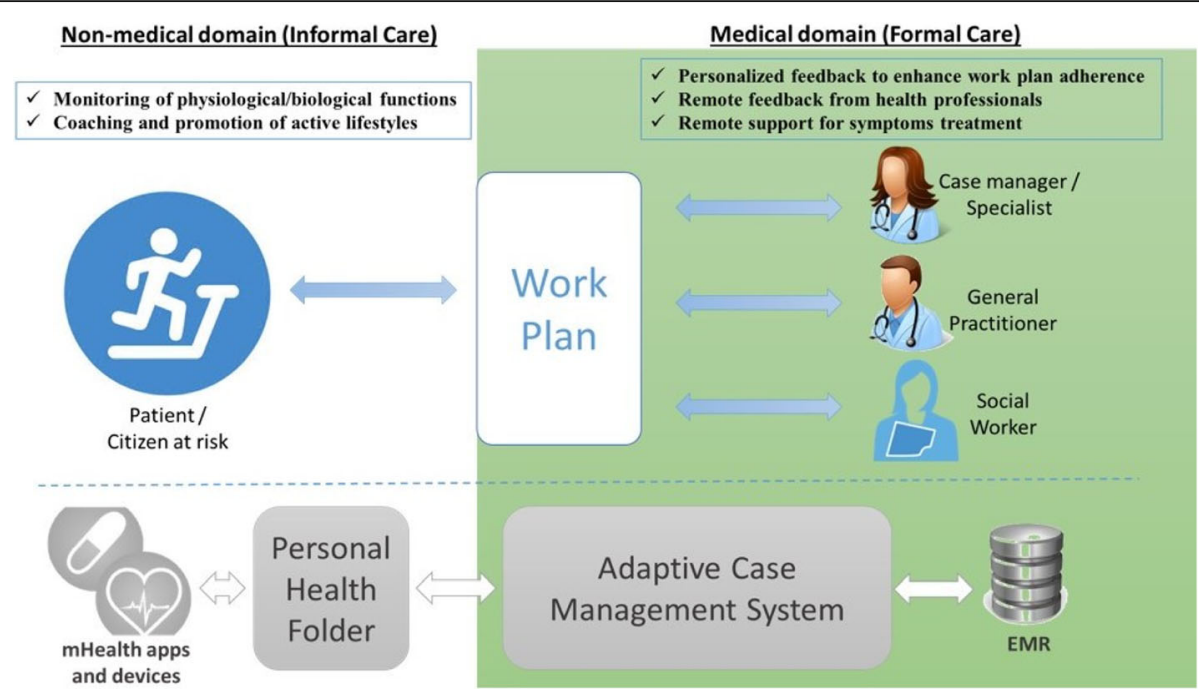

Fig. 1 Settings of the implementation studies. The figure shows two interoperable domains with technological elements providing support to the services promoting active lifestyles integrated within the action plan of the patient. On the left, Informal Care area with the patient having access to the Personal Health Folder (PHF) wherein she/he can answer questionnaires, perform monitoring through mHealth apps, and have access to a follow-up reports and tailored educational information, as defined in the work plan (center of the figure). On the right, the Formal Care domain wherein the case manager (physiotherapist) and/or general practitioner has access to an adaptive case management system for work plan prescription, follow-up and coaching. The adaptive case management system supports execution of the patient work plan and provides a bridge of interoperability and collaborative tools among the patient (through the PHF), the case manager and the electronic medical record (EMR)

the population-based risk stratification pyramid [27, 28], namely: i) Prehabilitation for high risk candidates to major surgery (Prehabilitation study); ii) Community-based rehabilitation and promotion of PA for clinical stable chronic patients with moderate to severe disease (Rehabilitation study); and, iii) Promotion of PA and healthy lifestyles for citizens at risk and patients with mild disease (Lifestyle study). The second aim is refinement and assessment of the innovative ICT-supporting tools alluded to above. Thirdly, the program adopts the Triple Aim approach $[29,30]$ for assessment of the three implementation studies and to select key performance indicators

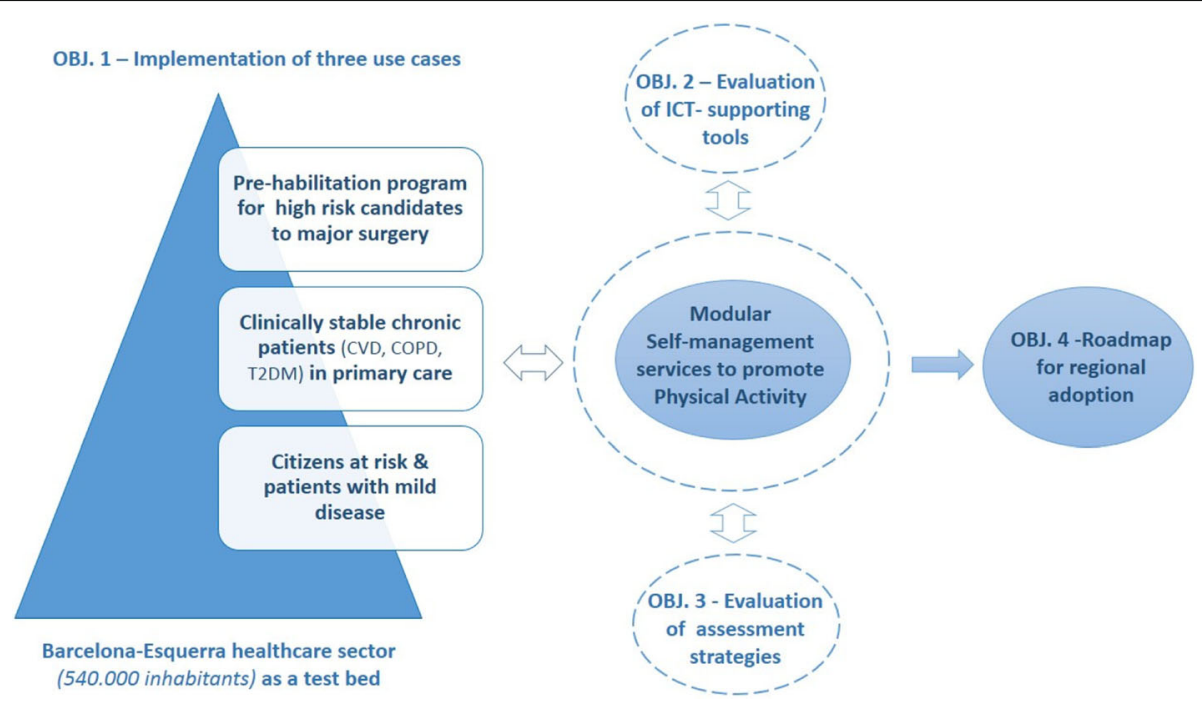

Fig. 2 Objectives of the implementation studies. The figure displays the four main objectives of the protocol considered as pivotal steps to achieve regional adoption of collaborative self-management services promoting physical activity across health-care tiers. Deployment of three use cases in one of the healthcare sectors of the city of Barcelona will target chronic obstructive pulmonary disease (COPD), cardiovascular disorders (CVD) and type 2 diabetes mellitus (T2DM). A patient-centered approach will be adopted. ICT stands for information and communication technologies 
(KPI) to be used for follow-up of the services beyond the implementation phase. Finally, the implementation studies will serve to generate a roadmap for regional adoption of the PA services. The sample size will grow according to the capacity of the service. The figures indicated below are realistic estimations.

\section{Specific aims of each of the three implementation studies}

A recent randomized controlled trial (RCT) carried out in high-risk candidates to major abdominal surgery has demonstrated high efficacy of prehabilitation reducing postoperative complications in these patients [31]. The main aim of the Prehabilitation study $(n=1000)$ in the current protocol is to assess cost-effectiveness of the deployment of the intervention as mainstream service at Hospital Clinic de Barcelona.

A previous non-randomized pilot study carried out by the research team [21] identified determinants of adoption of a community-based rehabilitation service for chronic patients, as well as its potential for health-value generation. The main objective of the Rehabilitation study $(n=800)$ is to assess both cost-effectiveness and sustainability of a community-based program combining exercise training and promotion of PA in clinically stable chronic patients showing one or more of the following target disorders: i) Chronic obstructive pulmonary disease (COPD) or other chronic respiratory conditions; ii) Cardiovascular disorders (CVD); and/or iii) Type II diabetes mellitus (T2DM).

Finally, the Lifestyle study $(n=1800)$ will assess large-scale deployment and sustainability of a portfolio of services promoting PA and healthy lifestyles for citizens at risk for the targeted chronic conditions and patients showing mild target disease(s). The study will also assess the impact of the intervention on health-related quality of life.

\section{Studies design and population}

The three implementation studies alluded to above aim at assessing cost-effectiveness and sustainability (business model) of the corresponding intervention as well as to identify factors modulating the success of large scale deployment. To this end, for each implementation study, an intervention group will be compared to a control group (usual care). Briefly, the Prehabilitation study will be deployed at Hospital Clinic de Barcelona following a quasi-experimental design using propensity score methods [32] wherein age, gender and population-based health-risk assessment are main matching variables between intervention and control groups. Adjusted Morbidity Groups (GMA) [33, 34] will be used for health risk scoring purposes. The Rehabilitation study will be deployed in the community selecting primary care centers of the healthcare sector as intervention or control units following an open cluster randomized control trial design with a 1:1 ratio. Finally, the Lifestyle study will be a community-based protocol following a quasi-experimental design, as described above.

Inclusion criteria for the Prehabilitation study are: i) Candidates to major elective abdominal, gynecological, cardiovascular, urological and thoracic surgical procedures; ii) Patients presenting high surgical risk defined by: age above 70 years and/or American Society of Anesthesiologists (ASA) score III/IV [35]; and, iii) A tentative surgical schedule allowing for at least 4 weeks for the prehabilitation intervention.

The Rehabilitation study has the following inclusion criteria: i) Patients suffering one or more targeted chronic conditions with moderate-to-severe disease of the main disorder; and, ii) Patients showing history of frequent exacerbations.

Finally, the inclusion criteria for the Lifestyle study are as follows: i) Citizens at risk for chronic conditions; and, ii) Patients showing mild target disease(s). All of them recruited through advertisements and through primary care centers.

Exclusion criteria for the three implementation studies are as follows: i) Emergency surgery (applicable only for the Prehabilitation study); ii) Unstable cardiac of respiratory disease; iii) Locomotor limitation precluding the practice of exercise; and, iv) Cognitive deterioration impeding the adherence to the program.

\section{Interventions}

The entry point to each program, as well as the core management and coordination functions, will be ascribed to the primary care team composed by general practitioner and case manager (physiotherapist). They will be directly in charge of the six common steps of the service workflow, namely: i) inclusion; ii) characterization and re-assessment of patient's work plan; iii) integration of the intervention into the patient's action plan; iv) execution of the specific program; v) follow-up/event handling; and, (vi) discharge from the program, as explained in detail in Section 1S.

\section{Prehabilitation study}

The current protocol aims to rollout the recently reported prehabilitation service [31] to all high-risk candidates to any major surgical procedure.

Candidates for the prehabilitation study fulfilling the inclusion criteria will be identified by the anesthesiologist in the pre-anesthesia visit. All candidates will be assessed in order to address a variety of issues: i) Identify the overall needs of the candidate; ii) Perform a baseline evaluation of his/her fitness and physical activity levels; and, iii) Identify his/her adherence profile, as well as factors and circumstances that 
may modulate practicalities of the intervention. Personalization of the prehabilitation plan involves the following main actions: i) Calendar and planning of face to face visits and remote (virtual) contacts with healthcare professionals; ii) Intensity/volume of the supervised endurance training program; iii) Threshold of minimum steps per day to promote physical activity; iv) Specific nutritional counseling and intervention (if malnutrition screening universal tool $\geq 2$ [36]); v) Group-oriented psychological interventions (mindfulness); and, vi) Integration of the prehabilitation intervention into the overall work-plan of the patient (see Additional file 1: section "Methods/design").

\section{Rehabilitation study}

Health value generation by personalized services including endurance training and promotion of PA will be assessed in chronic patients with target diseases. Briefly, the planned six-month period intervention will have two phases including, as a first step, reassessment of the patient work-plan aiming at optimization of both pharmacological and non-pharmacological therapies. The first phase of the program will start with a motivational interview wherein the intervention will be explained and co-designed with the patient. It will be followed by: i) Supervised endurance training sessions with a flexible duration of 2 to 6 weeks; ii) Promotion of active lifestyle;

Table 1 Main study outcomes of the implementation protocols

\begin{tabular}{|c|c|c|}
\hline Triple Aim & Outcome & Data source \& Instrument \\
\hline \multirow[t]{10}{*}{ Health and well-being } & Socio-demographics & Catalan Health Surveillance System \& Electronic Medical Records \\
\hline & Multi-morbidities & Catalan Health Surveillance System \& Electronic Medical Records \\
\hline & Patient Clinical Data & Electronic Medical Records \\
\hline & Health-related quality of life & SF12 questionnaire \\
\hline & $\begin{array}{l}\text { Healthy lifestyle (Tobacco/Nutrition/Alcohol/Physical } \\
\text { Activity) }\end{array}$ & Electronic Medical Records \\
\hline & Physical activity & Yale Physical Activity Survey \& Monitoring \\
\hline & Psychological well-being & Hospital Anxiety and Depression scale \\
\hline & Therapeutic plan (Pharmacological/Others) & Catalan Health Surveillance System \& Electronic Medical Records \\
\hline & $\begin{array}{l}\text { Intermediate outcomes (see costs): } \\
\text { - Emergency Department visits } \\
\text { - General Practitioner visits } \\
\text { - Cumulative days per year admitted in hospital } \\
\text { - Multiple drugs prescription } \\
\text { - Potentially avoidable hospitalizations } \\
\text { - Hospital readmissions } \\
\text { - Needs for social support }\end{array}$ & Catalan Health Surveillance System \\
\hline & Mortality & Catalan Health Surveillance System/Electronic Medical Records \\
\hline \multirow[t]{4}{*}{ Experience with care } & Use of the Personal Health Folder \& engagement & Catalan Health Surveillance System \\
\hline & Home-based technological support & Electronic Medical Records \\
\hline & Access to integrated care & Catalan Health Surveillance System \\
\hline & Patient satisfaction \& engagement & Electronic Medical Records (non-standard questionnaire) \\
\hline \multirow[t]{7}{*}{ Costs $^{\mathrm{a}}$} & Total health and social care cost & Catalan Health Surveillance System \\
\hline & Primary Care & Catalan Health Surveillance System \\
\hline & $\begin{array}{l}\text { Hospital-related Care } \\
\text { - Admissions } \\
\text { - Emergency Room consultations } \\
\text { - Outpatient specialized care }\end{array}$ & Catalan Health Surveillance System \\
\hline & Pharmacy & Catalan Health Surveillance System \\
\hline & Mental Health & Catalan Health Surveillance System \\
\hline & Socio-sanitary services & Catalan Health Surveillance System \\
\hline & $\begin{array}{l}\text { Other costs } \\
\text { - Respiratory therapies } \\
\text { - Dialysis } \\
\text { - Rehabilitation } \\
\text { - Non-urgent patient transport }\end{array}$ & Catalan Health Surveillance System \\
\hline
\end{tabular}

a The Catalan Health Surveillance System registries allow allocation of healthcare expenditure to each patient through the Personal Health Identification Number which facilitates analysis of total healthcare expenditure in complex patients 
and, iii) Patient empowerment for self-management of his/her condition aiming at increasing program adherence. The second phase of the intervention will include promotion of PA and self-management using the PHF [37] with remote off-line supervision by a case manager (see Additional file 1: Section 3).

\section{Lifestyle study}

The main objective is to assess the impact of a program promoting PA and healthy lifestyles in citizens at risk and patients with mild chronic disease. Briefly, key aspects of the intervention are: i) A motivational interview to personalize the intervention; ii) Familiarization of the candidate with the use of the PHF as a self-management tool; and, iii) Assignment of one case manager for off-line remote surveillance of the service. Moreover, the subject will receive information on a portfolio of optional services offered by the service (see Additional file 1: Section "Conclusions").

\section{Assessments}

The protocol evaluation will follow a Triple Aim approach $[29,30]$ considering pre-defined outcome variables for: i) Health and well-being; ii) Experience with care; and, iii) Costs (Table 1). Assessment will be carried out combining empirical questionnaire data collection, information from electronic medical records and registry data. The main study outcome will be twofold: i) Demonstration of cost-effectiveness of the interventions; and, ii) Identification of factors that modulate success of large-scale deployment. Moreover, an ancillary purpose of the implementation studies is to identify and evaluate key performance indicators (KPI) that could be used for long-term follow-up of the service at health system level. To this end, implementation research tools organized within the frame of the Model for ASsesment of Telemedicine applications (MAST) [38] will be considered. During the co-design phases, KPI will be identified and consolidated to be used for long-term assessment of the adoption process (Fig. 3). Most of the variables will be obtained from the automated Catalan Surveillance System [28].

\section{Regional deployment}

The fact that we are building-up on top of a rather mature setting for integrated care adoption reinforces the chances of successful regional deployment of the services. The use of a Plan-Do-Study-Act (PDSA) [34] methodology with iterative cycles shall consolidate refined services at the end of the first year and it will subsequently facilitate regional deployment in Catalonia (Fig. 3). As mentioned, assessment of KPI useful for long-term follow-up of the deployment beyond the implementation studies constitutes a key goal. A roadmap for regional deployment of the three interventions will be produced at the end of the implementation studies.

\section{Discussion}

The present protocol aims to evaluate the determinants of large-scale deployment and adoption of three novel collaborative self-management services promoting healthy lifestyles. We believe that the protocol represents a highly relevant step to foster synergies between non-pharmacological and pharmacological care paving the way for enhanced preventive care for chronic patients.

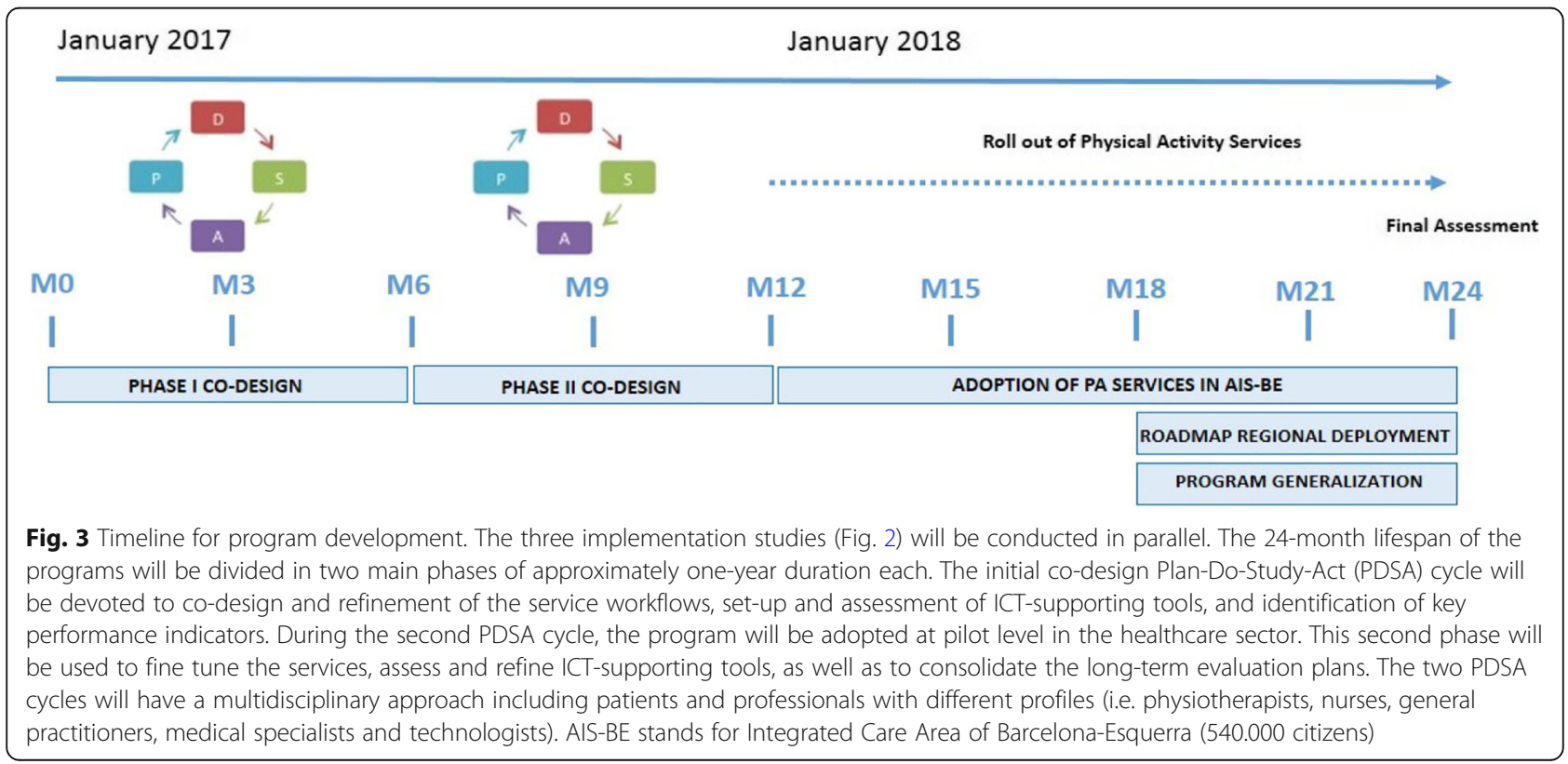


The study protocol adopts a population-health approach aiming at articulating a test bed that shall facilitate a successful rollout of the three implementation studies at regional level. Synergies across healthcare tiers will be fostered throughout the program deployment. For example, the current hospital-based prehabilitation study will be expanded and enriched with appropriate health-risk assessment tools aiming at enhancing perioperative risk prediction and care at community level. Thus, it should facilitate productive collaborative work between specialized and primary care professionals. Moreover, the program is expected to be highly transferable to other geographical areas.

\section{Conclusions}

The current manuscript reports on the protocol for regional deployment of collaborative self-management services promoting healthy lifestyles that will be carried out in Catalonia during the period 2017-2019.

\section{Additional file}

Additional file 1: Protocol for regional implementation of collaborative self-management services to promote physical activity. This file includes the specific details of each of the three protocols described in the main manuscript. (DOC $531 \mathrm{~kb}$ )

\section{Abbrevations}

ACM: Adaptive Case Management; AISBE: Area de Salut Barcelona-Esquerra; ASA: American Society of Anesthesiologists; COPD: Chronic Obstructive Pulmonary Disease; GMA: Adjusted Matching Groups; $\mathrm{HC}^{3}$ : Historia Clínica Compartida de Catalunya; ICT: Information and Communication Technologies; KPI: Key Performance Indicators; LMS: La Meva Salut; MAST: Model for ASsessment of Telemedicine; PA: Physical Activity; PDSA: Plan-Do-Study-Act; PHF: Personal Health Folder; RCT: Randomized Controlled Trial

\section{Acknowledgements}

We would like acknowledge the following projects: CONNECARE H2020689802, NEXTCARE COMRDI15-1-0016, ACT@Scale (HP-PJ-2015, 3HP, 709770) and Generalitat de Catalunya (2014SGR661).

\section{Funding}

This manuscript was supported by FIS-PITES (PI15/00576).

\section{Availability of data and materials}

The datasets used and/or analyzed during the current study are available from the corresponding author on reasonable request.

\section{Authors' contributions}

$A B G$ and JR contributed to the conception and design of this work, wrote the first draft of the paper and participated revising the manuscript; and are in agreement as to the integrity of the work and the contributions of all the authors. EGS, IB, IC, GMP, FB, FM, MC, SM, MS, AS, MU, JB, JV and MS, contributed to the conception and design of this work; participated in the writing and revising the manuscript; and are in agreement as to the integrity of the work and the contributions of all the authors. All authors have read and approved the final manuscript.

\section{Ethics approval and consent to participate}

The Ethics Committee for Clinical Research of Hospital Clínic de Barcelona approved the study protocol and the informed consent for the subjects (HCB/2016/0883).
Consent for publication

Not applicable.

\section{Competing interests}

The authors declare that they have no competing interests.

\section{Publisher's Note}

Springer Nature remains neutral with regard to jurisdictional claims in published maps and institutional affiliations.

\section{Author details}

${ }^{1}$ Respiratory Medicine Department, Hospital Clínic de Barcelona, CIBERES, Barcelona, Catalonia, Spain. ${ }^{2}$ Institut d'Investigacions Biomèdiques August Pi i Sunyer (IDIBAPS), Universitat de Barcelona, Barcelona, Catalonia, Spain. ${ }^{3}$ Anesthesiology Department, Hospital Clínic de Barcelona, Barcelona, Catalonia, Spain. ${ }^{4}$ EURECAT - Technological Center of Catalonia, Barcelona, Catalonia, Spain. ${ }^{5}$ Endocrinology and Nutrition Department, Hospital Clínic de Barcelona, IDIBAPS, University of Barcelona, Barcelona, Catalonia, Spain. ${ }^{6}$ Cardiology Department, Hospital Clínic de Barcelona, IDIBAPS, University of Barcelona, Barcelona, Catalonia, Spain. ${ }^{7}$ Consorci d'Atenció Primària de Salut Barcelona Esquerra (CAPSBE), Barcelona, Catalonia, Spain.

Received: 12 May 2017 Accepted: 5 July 2018

Published online: 17 July 2018

\section{References}

1. Kohl HW 3rd, Craig CL, Lambert EV, Inoue S, Alkandari JR, Leetongin G, et al. The pandemic of physical inactivity: global action for public health. Lancet. 2012;380(9838):294-305.

2. Lee IM, Shiroma EJ, Lobelo F, Puska P, Blair SN, Katzmarzyk PT. Effect of physical inactivity on major non-communicable diseases worldwide: an analysis of burden of disease and life expectancy. Lancet. 2012; 380(9838):219-29.

3. Watz H, Pitta F, Rochester CL, Garcia-Aymerich J, ZuWallack R, Troosters T, et al. An official European Respiratory Society statement on physical activity in COPD. Eur Resp J. 2014;44(6):1521-37.

4. Physical Activity Guidelines Advisory Committee report, 2008. To the Secretary of Health and Human Services. Part A: executive summary. Nutr Rev. 2009;67(2):114-20.

5. Sieverdes JC, Sui X, Lee DC, Church TS, McClain A, Hand GA, et al. Physical activity, cardiorespiratory fitness and the incidence of type 2 diabetes in a prospective study of men. Br J Sports Med. 2010;44(4):238-44.

6. Li S, Zhao JH, Luan J, Ekelund U, Luben RN, Khaw KT, et al. Physical activity attenuates the genetic predisposition to obesity in 20,000 men and women from EPIC-Norfolk prospective population study. PLoS Med. 2010;7(8). https://doi.org/10.1371/journal.pmed.1000332.

7. Friedenreich CM, Neilson HK, Lynch BM. State of the epidemiological evidence on physical activity and cancer prevention. Eur J Cancer. 2010;46(14):2593-604.

8. Mora S, Cook N, Buring JE, Ridker PM, Lee IM. Physical activity and reduced risk of cardiovascular events: potential mediating mechanisms. Circ. 2007;116(19):2110-8.

9. Manini TM, Everhart JE, Patel KV, Schoeller DA, Colbert LH, Visser M, et al. Daily activity energy expenditure and mortality among older adults. JAMA. 2006;296(2):171-9.

10. Rovio S, Kareholt I, Helkala EL, Viitanen M, Winblad B, Tuomilehto J, et al. Leisure-time physical activity at midlife and the risk of dementia and Alzheimer's disease. Lancet Neurol. 2005;4(11):705-11.

11. Heath GW, Parra DC, Sarmiento OL, Andersen LB, Owen N, Goenka S, et al, Evidence-based intervention in physical activity: lessons from around the world. Lancet. 2012;380(9838):272-81.

12. Spruit MA, Singh SJ, Garvey C, ZuWallack R, Nici L, Rochester C, et al. An official American Thoracic Society/European Respiratory Society statement: key concepts and advances in pulmonary rehabilitation. Am J Respir Crit Care Med. 2013;188(8):e13-64.

13. Ventura-Clapier R, Mettauer B, Bigard X. Beneficial effects of endurance training on cardiac and skeletal muscle energy metabolism in heart failure. Cardiovasc Res. 2007;73(1):10-8.

14. Strasser B, Pesta D. Resistance training for diabetes prevention and therapy: experimental findings and molecular mechanisms. Biomed Res Int. 2013;2013:805217. 
15. Pleguezuelos E, Perez ME, Guirao L, Samitier B, Ortega P, Vila X, et al. Improving physical activity in patients with COPD with urban walking circuits. Respir Med. 2013;107(12):1948-56.

16. Heppner PS, Morgan C, Kaplan RM, Ries AL. Regular walking and longterm maintenance of outcomes after pulmonary rehabilitation. J Cardiopul Rehabil. 2006;26(1):44-53.

17. Donesky-Cuenco D, Janson S, Neuhaus J, Neilands TB, Carrieri-Kohlman V. Adherence to a home-walking prescription in patients with chronic obstructive pulmonary disease. Heart Lung. 2007;36(5):348-63.

18. Moy ML, Weston NA, Wilson EJ, Hess ML, Richardson CR. A pilot study of an internet walking program and pedometer in COPD. Respir Med. 2012;106(9):1342-50.

19. Demeyer H, Louvaris Z, Frei A, Rabinovich RA, de Jong C, Gimeno-Santos E, Loeckx M, Buttery SC, Rubio N, Van der Molen T, Hopkinson NS, Vogiatzis I, Puhan MA, Garcia-Aymerich J, Polkey MI, Troosters T, Mr Papp PROactive study group and the PROactive consortium. Physical activity is increased by a 12week semiautomated telecoaching programme in patients with COPD: a multicentre randomised controlled trial. Thorax. 2017; [Epub ahead of print].

20. Whitehead $L$, Seaton $P$. The effectiveness of self-management mobile phone and tablet apps in long-term condition management: a systematic review. J Med Internet Res. 2016;18(5):e97.

21. Barberan-Garcia A, Vogiatzis I, Solberg HS, Vilaro J, Rodriguez DA, Garasen HM, et al. Effects and barriers to deployment of telehealth wellness programs for chronic patients across 3 European countries. Respir Med. 2014;108(4):628-37.

22. Department of Health, C. Catalonia Health Plan for 2016-2020 (in Catalan). (2016). Available at: http://salutweb.gencat.cat/web/.content/ home/el_departament/Pla_salut/pla_salut_2016_2020/Documents/Pla_ salut_Catalunya_2016_2020.pdf.

23. Cano I, Lluch-Ariet M, Gomez-Cabrero D, Maier D, Kalko S, Cascante M, Tegnér J, et al. Biomedical Research in a Digital Health Framework. BMC J Transl Med. 2014;12(Suppl 2):S10.

24. Regional health interoperability program in Catalonia (iS3). http://salutweb.gencat. cat/ca/ambits_tematics/inies_dactuacio/tecnologies_informacio_i_comunicacio/ is3/ and https://ticsalutsocial.cat/en/projects/observatory/is3-project/.

25. Marimon-Sunol S, Rovira-Barberà M, Acedo-Anta M, Nozal-Baldasjos MA, Guanyabens-Calvet J. Shared electronic health record in Catalonia. Med Clin (Barc). 2010;134(Suppl 1):45-8.

26. Cano I, Alonso A, Hernandez C, Burgos F, Barberan-Garcia A, Roldan J, Roca J. An adaptive case management system to support integrated care services: lessons learned from the NEXES project. J Biomed Inform. 2015;55:11-22.

27. Kindig D, Stoddart G. What is population health? Am J Public Health. 2003; 93:380-3.

28. Dueñas-Espín I, Vela E, Paus S, et al. Proposals for enhanced health risk assessment and stratification in an integrated care scenario. BMJ Open. 2016;6(4):e010301.

29. Berwick DM, Nolan TW, Whittington J. The triple aim: care, health, and cost. Health Aff. 2008:27:759-69.

30. Whittington JW, Nolan K, Lewis N, Torres T. Pursuing the triple aim: the first 7 years. Milbank Q. 2015;93:263-300.

31. Barberan-Garcia A, Ubré M, Roca J, Lacy AM, Burgos F, Risco R, et al. Personalised prehabilitation in high-risk patients undergoing elective major abdominal surgery: a randomized double-blind controlled trial. Ann Surg. 2017: https://doi.org/10.1097/SLA.0000000000002293.

32. Austin PC. An introduction to propensity score methods for reducing the effects of confounding in observational studies. Multivariate Behav Res. 2011;46:399-424.

33. SELFIE. Sustainable intEgrated care modeLs for multi-morbidity: delivery, Flnancing and performancE. Project no 634288 Available at: http://www.selfie2020.eu/

34. Taylor MJ, McNicholas C, Nicolay C, Darzi A, Bell D, et al. Systematic review of the application of the plan-do-study-act method to improve quality in healthcare. BMJ Qual Saf. 2014;23:290-8.

35. 2016 Relative value guide book: A guide for anesthesia values. Washington D.C: American Society of Anesthesiologists; 2015.

36. Malnutrition Advisory Group, a Standing Committee of BAPEN. Malnutrition Universal Screening Tool. May 2003 (review August 2011). Available at: http://www.bapen.org.uk/pdfs/must/must_full.pdf. Accessed 16 Nov 2016.

37. Generalitat de Catalunya. Cat@Salut La Meva Salut. Canal personal de salut. at http://catsalut.gencat.cat/ca/serveis-sanitaris/la-meva-salut/.

38. Kidholm K, Ekeland AG, Jensen LK, Rasmussen J, Pedersen CD, Bowes A, et al. A model for Assessment of telemedicine applications: Mast. Int J Technol Assess. 2012;28(1):44-51.

Ready to submit your research? Choose BMC and benefit from:

- fast, convenient online submission

- thorough peer review by experienced researchers in your field

- rapid publication on acceptance

- support for research data, including large and complex data types

- gold Open Access which fosters wider collaboration and increased citations

- maximum visibility for your research: over $100 \mathrm{M}$ website views per year

At $\mathrm{BMC}$, research is always in progress.

Learn more biomedcentral.com/submissions 